\title{
@creative
}

\section{On the anti-Kekulé problem of cubic graphs*}

\author{
Qiuli Li \\ School of Mathematics and Statistics, Lanzhou University, \\ Lanzhou, Gansu 730000, China \\ Wai Chee Shiu ${ }^{\dagger}$, Pak Kiu Sun \\ Department of Mathematics, Hong Kong Baptist University, \\ Kowloon Tong, Hong Kong, China \\ Dong Ye \\ Department of Mathematical Sciences, Middle Tennessee State University, \\ Murfreesboro, TN 37132, United States
}

Received 6 September 2016, accepted 8 July 2018, published online 12 August 2018

\begin{abstract}
An edge set $S$ of a connected graph $G$ is called an anti-Kekulé set if $G-S$ is connected and has no perfect matchings, where $G-S$ denotes the subgraph obtained by deleting all edges in $S$ from $G$. The anti-Kekulé number of a graph $G$, denoted by $a k(G)$, is the cardinality of a smallest anti-Kekulé set of $G$. It is NP-complete to determine the antiKekulé number of a graph. In this paper, we show that the anti-Kekulé number of a 2connected cubic graph is either 3 or 4 , and the anti-Kekulé number of a connected cubic bipartite graph is always equal to 4 . Furthermore, a polynomial time algorithm is given to find all smallest anti-Kekulé sets of a connected cubic graph.
\end{abstract}

Keywords: Anti-Kekulé set, anti-Kekulé number, cubic graphs.

Math. Subj. Class.: 05C10, 05C70, 05C90

*This work is supported by NSFC (grant nos. 11401279 and 11371180), the Specialized Research Fund for the Doctoral Program of Higher Education (No. 20130211120008), the Fundamental Research Funds for the Central Universities (no. lzujbky-2017-28), and General Research Fund of Hong Kong.

${ }^{\dagger}$ The corresponding author.

E-mail addresses: qlli@lzu.edu.cn (Qiuli Li), wcshiu@hkbu.edu.hk (Wai Chee Shiu), lionel@hkbu.edu.hk (Pak Kiu Sun), dong.ye@mtsu.edu (Dong Ye) 


\section{Introduction}

Let $G$ be a graph. A perfect matching of a graph $G$ is a set of non-adjacent edges that covers all vertices of $G$. A perfect matching of a graph is also called a Kekulé structure in mathematical chemistry and statistical physics. An edge set $S$ of a connected graph $G$ is called an anti-Kekulé set if $G-S$ is connected and has no perfect matchings, where $G-S$ denotes the subgraph obtained by deleting all edges in $S$ from $G$. The anti-Kekulé number of a graph $G$, denoted by $a k(G)$, is the cardinality of a smallest anti-Kekule set of $G$. The anti-Kekule number of a graph is hard to be determined in general. It has been proved recently that it is an NP-complete problem to determine the anti-Kekule number of a connected bipartite graph by Lü, Li and Zhang [17].

In chemistry and physics, graphs are used to represent the skeletons of molecules, and Kekulé structures (or perfect matchings) are used to model special structures of bonds between atoms. For example, for a benzenoid hydrocarbons, graphens or fullerenes, a Kekulé structure of these molecules stands for double bonds between atoms. An antiKekule set is a set of double bonds whose removal significantly affects the whole molecule structure by the valence bond (VB) theory (cf. [15]).

A fullerene is a 3-connected plane cubic graph such that every face is either a hexagon or a pentagon. For example, $\mathrm{C}_{60}$ is a fullerene with 60 vertices such that all pentagons are disjoint. The anti-Kekulé number of $\mathrm{C}_{60}$ is proved to be 4 by Vukičević [26]. A leapfrog fullerene is a fullerene obtained by the leapfrog operation (see [16]). Kutnar et al. [16] obtained a bound for the anti-Kekule number of leapfrog fullerenes as follows.

Theorem 1.1 ([16]). Let $G$ be a leapfrog fullerene. Then $3 \leq a k(G) \leq 4$.

The above result was improved by Yang et al. [28] by proving that all fullerenes have anti-Kekulé number 4 . The anti-Kekulé numbers of other interesting graphs, such as benzenoid hydrocarbons [2, 27], fence graphs [22], infinite triangular, rectangular and hexagonal grids [25] as well as cata-condensed phenylenes [35], have been investigated.

The result on fullerenes has been generalized to general cubic graphs with high cyclic edge-connectivity in [30]. A graph $G$ is cyclically $k$-edge-connected if $G$ cannot be separated into two components, each containing a cycle, by deletion of fewer than $k$ edges. The cyclic edge-connectivity $c \lambda(G)$ of a graph $G$ is the maximum $k$ such that $G$ is cyclically $k$-edge-connected. An edge set $S$ is called an odd cycle edge-transversal of a graph $G$ if $G-S$ is bipartite. The size of a smallest odd cycle edge-transversal of $G$ is denoted by $\tau_{\text {odd }}(G)$.

Theorem 1.2 ([30]). Let $G$ be a cyclically 4-edge-connected cubic graph. Then either $a k(G)=4$ or $1 \leq \tau_{\text {odd }}(G) \leq 3$.

The result above can be used to determine the anti-Kekulé number of fullerenes. Since a smallest odd cycle-transversal of a fullerene graph contains at least 6 edges and the cyclic edge-connectivity of a fullerene graph is 5 (see [5, 20]), Theorem 1.2 implies that every fullerene has anti-Kekulé number 4 . However, Theorem 1.2 is not applicable to determine the anti-Kekulé numbers of some interesting graphs, such as, some boron-nitrogen fullerenes with low cyclic edge-connectivity, $(3,6)$-fullerenes etc.

A $(k, 6)$-cage $(k \geq 3)$ is a 3-connected cubic plane graph whose faces are either $k$-gons or hexagons. Došlić [5] shows that $(k, 6)$-cages only exist for $k=3,4$ and 5 . A fullerene is a $(5,6)$-cage and the $(4,6)$-cages and $(3,6)$-cages are usually called $(4,6)$-fullerenes (or 
boron-nitrogen fullerens) and (3,6)-fullerenes, respectively. Many researches have investigated the properties of these graphs in both mathematics and chemistry, such as hamiltonicity [9, 10], resonance [29, 31, 33], the forcing matching number [13, 34], and energy spectra of $(3,6)$-fullerenes $[4,14]$ which determines their electronic and magnetic properties $[3,21]$.

The cyclic edge-connectivity of $(k, 6)$-cages has been obtained by Došlić in [5]. Let $\mathcal{T}$ be a family of $(4,6)$-fullerenes, which consists of a tube with $n$ layers of hexagons (i.e. each layer is a cyclic chain of three hexagons) capped on both ends by a cap formed by three quadrangles.

Theorem 1.3 ([5]). Let $G$ be a $(k, 6)$-cage. Then $c \lambda(G)=3$ if $G \in \mathcal{T}$, and $c \lambda(G)=k$ otherwise.

In this paper, we consider the anti-Kekulé number of connected cubic graphs including those with low cyclic edge-connectivity. The following is our first major result.

Theorem 1.4. If $G$ is a 2-connected cubic graph, then $3 \leq a k(G) \leq 4$.

Since a leapfrog fullerene is 3 -connected, Theorem 1.1 is a direct corollary of Theorem 1.4. For bipartite cubic graphs, the result can be strengthened as follows.

Theorem 1.5. If $G$ is a connected cubic bipartite graph, then ak $(G)=4$.

Theorems 1.4 and 1.5 can be applied to determine the anti-Kekulé numbers of boronnitrogen fullerenes, $(3,6)$-fullerenes, toroidal and bipartite Klein-bottle fullerenes (see Section 3 for details). Based on Theorems 1.4 and 1.5, a polynomial time algorithm is given to find all smallest anti-Kekulé sets of a connected cubic graph $G$ in Section 4.

\section{Proofs of main results}

The well-known theorem of Tutte is essential to our proof of the main results.

Theorem 2.1 (Tutte's Theorem [23]). A graph $G$ has a perfect matching if and only if $c_{o}(G-U) \leq|U|$ for any $U \subseteq V(G)$, where $c_{o}(G-U)$ is the number of odd components of $G-U$.

A bridge is an edge of a connected graph whose deletion disconnects the graph. By Petersen's Theorem [19], every cubic graph without bridges has a perfect matching. Therefore, the anti-Kekule number of a 2-connected cubic graph $G$ (note that a cubic graph possesses the same connectivity and edge-connectivity) is at least one, that is, $a k(G) \geq 1$. Indeed, this lower bound can be improved and we present the proof by using Tutte's Theorem. For $X \subseteq V(G)$, let $\partial(X)$ denote the set of edges with one end in $X$ and the other end in $V(G)-X$. We also denote $d(X)=|\partial(X)|$.

Proof of Theorem 1.4. Let $A$ be an anti-Kekulé set of size $a k(G)$. According to the definition, $G^{\prime}:=G-A$ has no perfect matchings. Hence, Theorem 2.1 implies that there exists $S \subseteq V\left(G^{\prime}\right)$ such that $c_{o}\left(G^{\prime}-S\right)>|S|$. Choose such an $S$ with the maximum size.

Claim 1. $G^{\prime}-S$ has no even components and it has exactly $|S|+2$ odd components.

Suppose by the contrary, $G^{\prime}-S$ has an even component $H$. Thus, for any given vertex $v \in V(H), H-\{v\}$ has at least one odd component. Let $S^{\prime}=S \cup\{v\}$. Then $G^{\prime}-S^{\prime}$ has at least $c_{o}\left(G^{\prime}-S\right)+1$ odd components. That is,

$$
c_{o}\left(G^{\prime}-S^{\prime}\right) \geq c_{o}\left(G^{\prime}-S\right)+1>|S|+1=\left|S^{\prime}\right|,
$$


contradicting the choice of $S$. Therefore $G^{\prime}-S$ has no even component.

Since $G$ is a cubic graph, it has an even number of vertices. This implies that $c_{o}\left(G^{\prime}-S\right)$ and $|S|$ are of the same parity, thus $c_{o}\left(G^{\prime}-S\right) \geq|S|+2$. For any edge $e \in A$, since $A$ is an anti-Kekulé set with the smallest cardinality, $G^{\prime}+e$ has a perfect matching (note that $G^{\prime}+e$ is the graph with vertex set $V\left(G^{\prime}\right)$ and edge set $\left.E\left(G^{\prime}\right) \cup\{e\}\right)$. Hence $c_{o}\left(G^{\prime}+e-S\right) \leq|S|$ by Theorem 2.1. Moreover, adding any edge $e$ to $G^{\prime}-S$ will connect at most two odd components. Therefore, $|S| \geq c_{o}\left(G^{\prime}+e-S\right) \geq c_{o}\left(G^{\prime}-S\right)-2 \geq|S|$ and thus, $c_{o}\left(G^{\prime}-S\right)-2=|S|$.

Claim 2. Let $G_{i}$, with $1 \leq i \leq|S|+2$, be the odd components of $G^{\prime}-S$. We have

$$
\sum_{i=1}^{|S|+2} d\left(G_{i}\right)-2 a k(G) \leq 3|S| .
$$

We count the number of edges between $S$ and the odd components, which is denoted by $N$, in two different ways. On one hand, $S$ contributes at most $3|S|$ to $N$. On the other hand, all the odd components send out $\sum_{i=1}^{|S|+2} d\left(G_{i}\right)-2 a k(G)$ edges to $N$. Thus $\sum_{i=1}^{|S|+2} d\left(G_{i}\right)-2 a k(G)=N \leq 3|S|$ and the claim holds.

Since $G$ is 2-edge-connected, $d\left(G_{i}\right) \geq 2$ for every $i$. By a simple computation $d\left(G_{i}\right)=$ $3\left|V\left(G_{i}\right)\right|-2\left|E\left(G_{i}\right)\right|$, which implies that $d\left(G_{i}\right)$ and $\left|V\left(G_{i}\right)\right|$ are of the same parity. Since every $G_{i}$ is an odd component, $\left|V\left(G_{i}\right)\right|$ is odd and hence $d\left(G_{i}\right)$ is odd, therefore $d\left(G_{i}\right) \geq 3$.

Substituting this inequality into Equation (2.1), we have

$$
3(|S|+2)-2 a k(G) \leq \sum_{i=1}^{|S|+2} d\left(G_{i}\right)-2 a k(G) \leq 3|S|,
$$

and so $a k(G) \geq 3$.

Now we are going to establish an upper bound on $a k(G)$ and it is sufficient to find an anti-Kekulé set of size 4 . Let $a \in V(G)$ and let $b$ as well as $c$ be its two distinct neighbors. Denote the two edges incident with $b$ other than $a b$ by $e_{1}$ and $e_{2}$, similarly, denote the two edges incident with $c$ other than $a c$ by $e_{3}$ and $e_{4}$. Hence, removing $E_{a}=\left\{e_{1}, e_{2}, e_{3}, e_{4}\right\}$ from $G$ will obtain a subgraph without perfect matchings. Therefore, $E_{a}$ is an anti-Kekulé set if $G-E_{a}$ is connected (there exists some vertex $a$ such that $G-E_{a}$ is not connected, see Figure 1). Consider the following two cases according to the different connectivities.

Case 1. G is 3-connected.

We are going to prove that for any vertex $a$ in $G, G-E_{a}$ is connected. Suppose by the contrary that $G-E_{a}$ is not connected. Then the vertices of $G$ are divided into two parts $X$ and $\bar{X}$ with a subset $E^{\prime} \subseteq E_{a}$ connecting them. Since $G$ is 3-connected, $E^{\prime}$ consists of three or four edges in $E_{a}$. If it contains three edges, by symmetry, we assume $E^{\prime}=\left\{e_{1}, e_{2}, e_{3}\right\}$. Since there are exactly three edges between $X$ and $\bar{X}$, the edge $a b$ lies in the same part. We may assume that both $a$ and $b$ belong to $X$. Since $\left\{a b, e_{3}\right\}$ divides $G$ into two parts $\bar{X} \cup\{b\}$ and $X \backslash\{b\},\left\{a b, e_{3}\right\}$ is a 2-edge-cut of $G$ which is a contradiction (note that a 2-edge-cut is an edge-cut of size 2). If $E^{\prime}$ contains four edges, that is $E^{\prime}=E_{a}=\left\{e_{1}, e_{2}, e_{3}, e_{4}\right\}$, then by a similar argument as above, we know that $a, b$ and $c$ lie in the same part, and moreover $\{a b, a c\}$ forms a 2-edge-cut of $G$ which is a contradiction. 


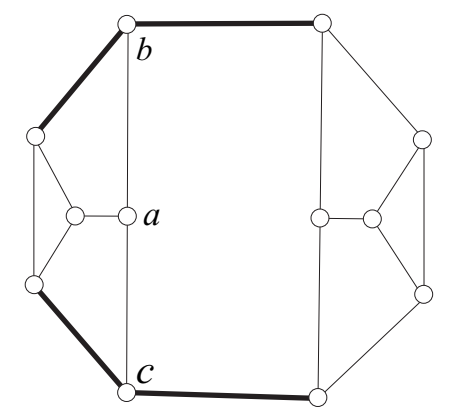

Figure 1: $E_{a}$ are the bold edges.

Note that $G-E_{a}$ is connected and $G-E_{a}$ has no perfect matchings. Therefore, $E_{a}$ is an anti-Kekulé set of size 4 and we have $a k(G) \leq 4$.

Case 2. G has connectivity 2.

Since $G$ is 2 -connected but not 3 -connected, there exist 2-edge-cuts. Moreover, each 2-edge-cut is an independent set, otherwise the third edge incident to their common end vertex is a bridge, which contradicts that $G$ is 2-connected. Every 2-edge-cut will split $G$ into exactly two subgraphs. Among those subgraphs, denote the subgraph with smallest cardinality by $G^{\prime}$ and the corresponding 2-edge-cut by $E=\left\{e_{4}, e_{5}\right\}$. Also, denote the end-vertices of $e_{4}$ and $e_{5}$ in $G^{\prime}$ by $v$ and $u$, respectively. Moreover, let $G^{\prime \prime}$ be the other subgraph obtained by deleting $E$.

Claim 3. $u v \notin E(G)$.

Assume $u v \in E(G)$. Then the edges incident with $u$ or $v$ other than $u v, e_{4}$ and $e_{5}$ form a 2-edge-cut. The deletion of this 2-edge-cut creates a subgraph with cardinality smaller than $G^{\prime}$, contradicting the choice of $E$.

Claim 4. No 2-edge-cut of $G$ contains an edge $e \in E\left(G^{\prime}\right)$.

Suppose the claim is false and there exists $e \in E\left(G^{\prime}\right)$ that lies in some 2-edge-cut $E^{\prime}=\left\{e, e^{\prime}\right\}$. No matter where $e^{\prime}$ lies in, the subgraph induced by $V\left(G^{\prime \prime}\right) \cup\{u\}$ or $V\left(G^{\prime \prime}\right) \cup\{v\}$ belongs to a component created by the deletion of $E^{\prime}$. Thus the cardinality of the other component is smaller than $G^{\prime}$, which contradicts the choice of $E$ and the claim holds.

Let $s$ be a neighbor of $v$ in $G^{\prime}$. Since $s$ is of degree 3 , there exists a neighbor $t(\neq u)$ of it in $G^{\prime}$. Let $e_{1}$ and $e_{2}$ be two incident edges of $t$ other than $s t$, and let $e_{3}$ be the edge incident with $v$ other than $s v$ and $e_{2}$. We claim that $\left\{e_{1}, e_{2}, e_{3}, e_{4}\right\}$ is an anti-Kekulé set. It is obvious that $G-\left\{e_{1}, e_{2}, e_{3}, e_{4}\right\}$ has no perfect matchings. If $G-\left\{e_{1}, e_{2}, e_{3}, e_{4}\right\}$ is not connected, then, similar to Case 1 , we obtain a 2 -edge-cut containing at least one edge in $G^{\prime}$. This is a contradiction and completes the proof.

The condition "2-edge-connected" in Theorem 1.4 is necessary because there exist cubic graphs with bridges and their anti-Kekule number is less than 3 (see Figure 2). More precisely, we have the following result.

Theorem 2.2. If $G$ is a connected cubic graph with bridges, then $a k(G) \leq 2$. 
Proof. Choose a bridge such that the deletion of it will give a subgraph with the smallest cardinality, we denote this subgraph by $G^{\prime}$ and the corresponding bridge by $e$. Let the end vertex of $e$ in $G^{\prime}$ be $u$ and let $v$ be a neighbor of $u$ in $G^{\prime}$. Moreover, let the two other edges incident with $v$ other than $u v$ be $e_{1}$ and $e_{2}$. Similar to the proof of Case 2 in Theorem 1.4, we have $G-\left\{e_{1}, e_{2}\right\}$ is connected. Since any bridge separates $G$ into two odd components, any perfect matching $M$ of $G$ should contain $e$. Also, $M$ contains one edge in $\left\{e_{1}, e_{2}\right\}$ and thus, $G-\left\{e_{1}, e_{2}\right\}$ has no perfect matchings. As a result, $\left\{e_{1}, e_{2}\right\}$ is an anti-Kekulé set and so $a k(G) \leq 2$.

Figure 2 presents three cubic graphs with anti-Kekulé numbers 0, 1 and 2, and the sets of bold edges denote their smallest anti-Kekulé sets respectively.
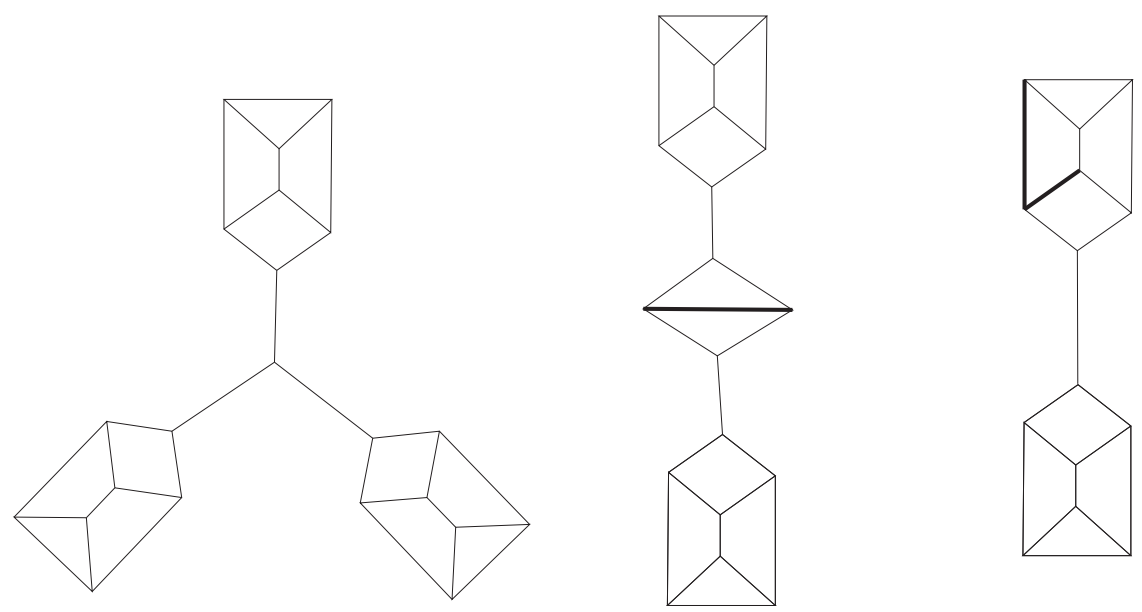

Figure 2: Three cubic graphs with anti-Kekulé numbers 0,1 and 2 respectively.

If the graphs being considered are bipartite, then a stronger result can be obtained by using Hall's Theorem.

Theorem 2.3 (Hall's Theorem [11]). Let $G$ be a bipartite graph with bipartition $W$ and $B$. Then $G$ has a perfect matching if and only if $|W|=|B|$ and for any $U \subseteq W,|N(U)| \geq|U|$ holds.

Proof of Theorem 1.5. First we show that a connected cubic bipartite graph is essentially 2-connected. By Theorem 2.3, a $k$-regular bipartite graph contains a perfect matching. Removing that perfect matching will result in a $(k-1)$-regular bipartite graph, and the same argument can be applied repeatedly. Finally, we deduce that a $k$-regular bipartite graph can be decomposed into $k$ disjoint perfect matchings. Since $G$ is a cubic bipartite graph, it can be decomposed into three disjoint perfect matchings $M_{1}, M_{2}$ and $M_{3}$, that is, $E(G)=M_{1} \cup M_{2} \cup M_{3}$. For any $e \in E(G)$, without loss of generality, let $e \in M_{1}$. Since $M_{1}$ and $M_{2}$ are disjoint perfect matchings of $G, M_{1} \cup M_{2}$ consists of disjoint even cycles and $e$ lies in one of them. Hence $e$ is not a bridge and $G$ is 2-edge-connected. Furthermore, a 2-edge-connected cubic graph is 2-connected, thus $G$ is 2-connected.

According to Theorem 1.4, we have $3 \leq a k(G) \leq 4$. Suppose by the contrary that $a k(G) \neq 4$, that is, $a k(G)=3$. Let $A=\left\{e_{1}, e_{2}, e_{3}\right\}$ be an anti-Kekulé set. Then $G-A$ 
has no perfect matchings. Assume $W$ and $B$ are the bipartition of $G$. According to Hall's theorem, there exists $S \subseteq W$ such that

$$
\left|N_{G-A}(S)\right| \leq|S|-1
$$

On the other hand, since $A$ is an anti-Kekule set with the smallest cardinality, we have

$$
|S| \leq\left|N_{G-A+e_{i}}(S)\right|
$$

for $i=1,2$ and 3 . Adding an edge $e_{i}$ to $G-A$ will increase the neighbors of $S$ by one (at most). Hence

$$
\left|N_{G-A+e_{i}}(S)\right| \leq\left|N_{G-A}(S)\right|+1 .
$$

Combining inequalities (2.2), (2.3) and (2.4), we obtain $|S|=\left|N_{G-A}(S)\right|+1$. Let $S^{\prime}=$ $N_{G-A}(S)$. The edges going out from $S$ are divided into two parts: either goes into $A$ or goes into $S^{\prime}$. Thus the number of edges between $S$ and $S^{\prime}$ is $3|S|-3$. Since $\left|S^{\prime}\right|=|S|-1$, there is no edge between $S^{\prime}$ and $W-S$. Therefore, $A$ is an edge-cut, which contradicts the definition of anti-Kekulé set and the proof is complete.

\section{Applications}

In this section, we apply Theorems 1.4 and 1.5 to obtain the anti-Kekulé numbers of several families of interesting graphs, such as boron-nitrogen fullerenes and $(3,6)$-fullerenes.

Theorem 3.1. If $G$ is a $(4,6)$-fullerene, then $a k(G)=4$.

Proof. Since $G$ is bipartite, the result follows immediately by Theorem 1.5.

Note that there are two classes of boron-nitrogen fullerenes, one with cyclic edgeconnectivity 3 and the other with cyclic edge-connectivity 4 . The anti-Kekulé number of the latter can be obtained by Theorem 1.2. Now we are going to determine the anti-Kekulé number of $(3,6)$-fullerenes and the following lemma is required. A cyclic 3-edge-cut of a $(3,6)$-fullerene is called trivial if it is formed by the edges incident to a triangle in common. A 3-edge-cut is called trivial if they are incident to a common vertex. Let $T_{n}(n \geq 1)$ be the graph consisting of $n$ concentric layers of hexagons in which each layer is a cyclic chain of two hexagons, capped on each end by a cap formed by two adjacent triangles (see Figure 3).

\section{Lemma 3.2 ([29]).}

(i) Every cyclic 3-edge-cut of a (3,6)-fullerene with connectivity 3 is trivial.

(ii) The connectivity of a $(3,6)$-fullerene is 2 if and only if it is isomorphic to $T_{n}$ for some $n \geq 1$.

Theorem 3.3. If $G$ is a $(3,6)$-fullerene, then $a k(G)=3$.

Proof. Let $G$ be a $(3,6)$-fullerene. Note that a $(3,6)$-fullerene has connectivity either 2 or 3 . Thus, Theorem 1.4 implies that $3 \leq a k(G) \leq 4$. To show that $a k(G)=3$, it suffices to give an anti-Kekulé set of size 3 .

First, assume that the connectivity of $G$ is 2 . By Lemma 3.2, $G$ has two triangles sharing a common edge. Let $S$ be the edge set of such a triangle (see Figure 3). Then 


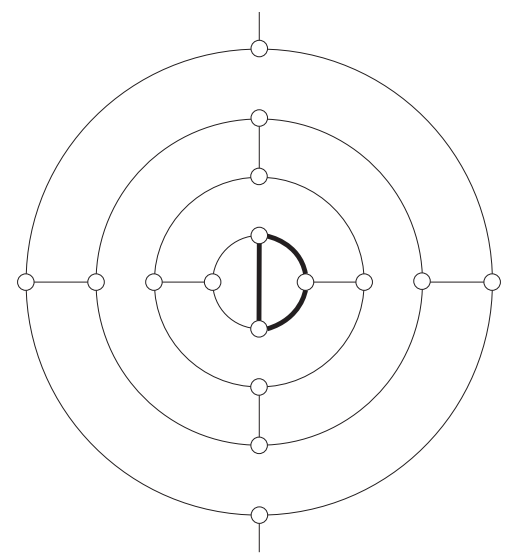

Figure 3: A $(3,6)$-fullerene $T_{3}$ and the set of bold edges form an anti-Kekulé set of it.

$G-S$ has two vertices of degree 1 adjacent to a common vertex. Hence $G-S$ has no perfect matching. Clearly, $G-S$ is connected. So $S$ is an anti-Kekulé set of size 3 .

In the following, assume that $G$ is 3 -connected. Let $S$ be a 3-edge-cut and let $G_{1}$ as well as $G_{2}$ be the two components of $G-S$. If $S$ is not trivial, then $d\left(G_{i}\right)=3$ and $\left|V\left(G_{i}\right)\right| \geq 2$ for $i=1,2$. Since $\left|V\left(G_{i}\right)\right|$ and $d\left(G_{i}\right)$ are of the same parity, it follows that $\left|V\left(G_{i}\right)\right| \geq 3$. Hence

$$
\left|E\left(G_{i}\right)\right|-\left|V\left(G_{i}\right)\right|=\frac{3\left|V\left(G_{i}\right)\right|-3}{2}-\left|V\left(G_{i}\right)\right|=\frac{\left|V\left(G_{i}\right)\right|-3}{2} \geq 0 .
$$

Therefore both $G_{1}$ and $G_{2}$ contain cycles. So $S$ is a cyclic 3-edge-cut. By Lemma 3.2, $S$ is a trivial cyclic 3-edge-cut. Hence, a 3-edge-cut of $G$ is either a trivial 3-edge-cut or a trivial cyclic 3 -edge-cut.

Let $a b c$ be a triangle of $G$. Let $e_{1}$ be the edge incident with $a$ but not inside of the triangle, and $e_{2}$ be the edge incident with $c$ but not contained in the triangle. The edge set $S=\left\{e_{1}, e_{2}, a c\right\}$ does not isolate a vertex or a triangle. So $S$ is not an edge-cut. In the subgraph $G-S$, both $a$ and $c$ have degree 1 and both of them are adjacent to $b$. So $G-S$ has no perfect matching. Therefore, $S$ is an anti-Kekulé set. This completes the proof.

Furthermore, since a toroidal fullerene or a bipartite Klein-bottle fullerene, whose definitions can be found in [32], is a cubic bipartite graph, the following result is a direct consequence of Theorem 1.5. Note that this result can also be deduced from Theorem 1.2.

Corollary 3.4. If $G$ is either a toroidal fullerene or a bipartite Klein-bottle fullerene, then $a k(G)=4$.

\section{Finding all the smallest anti-Kekulé sets}

The anti-Kekulé problem of graphs can be stated as follows.

Instance: A nonempty graph $G=(V, E)$ having a perfect matching and a positive $k$.

Question: Is there a subset $B \subseteq E$ with $|B| \leq k$ such that $G^{\prime}=(V, E \backslash B)$ is connected and $G^{\prime}$ has no Kekulé structure? 
In [17], the authors showed that anti-Kekulé problem on bipartite graphs is NP-complete. So it is hard to find a smallest anti-Kekule set of a given graph. However, for cubic graphs, the problem becomes much easier by Theorems 1.4 and 1.5: all the smallest anti-Kekulé sets of a cubic graph can be found in polynomial time. The algorithm finding all smallest anti-Kekulé sets $S$ of a cubic graph $G$ depends on how to find a maximum matching in the graph $G-S$. If the maximum matching of $G-S$ has size exactly $n / 2$ where $n$ is the number of vertices of $G$, then it is a perfect matching of $G-S$.

For a given graph $G$ with $n$ vertices, Edmonds [6] found an algorithm to find a maximum matching of $G$ in $O\left(n^{4}\right)$ steps, which is the blossom algorithm. An efficient implementation of Edmonds' algorithm takes $O\left(n^{3}\right)$ steps to find a maximum matching [7]. For bipartite graphs, Hopcroft and Karp [12] gave an algorithm taking $O\left(n^{5 / 2}\right)$ steps to find a maximum matching. Later, Micali and Vazirani [18, 24], Gabow and Tarjan [8], and Blum [1] have given algorithms to find a maximum matching of $G$ in $O(\sqrt{n} m)$ steps, where $m$ is the number of edges of $G$.

Theorem 4.1 ([1, 8, 18]). Let $G$ be a graph with $n$ vertices and $m$ edges. It takes $O(\sqrt{n} m)$ steps to find a maximum matching of $G$.

The connectedness of a graph $G$ with $n$ vertices can be determined by the breadthfirst search (BFS) algorithm, which takes $O(n)$ steps. Based on Theorems 1.4 and 1.5, by applying the BFS algorithm and the maximum matching algorithm to $G-S$, we can find all smallest anti-Kekulé sets $S$ of a cubic graph $G$.

Algorithm (Finding all smallest anti-Kekulé sets)

Input: A cubic graph $G$ with $n$ vertices.

Output: All the smallest anti-kekulé sets of $G$.

Step 1. Let $k=0$. Use the maximum matching algorithm on $G$. If $G$ has a maximum matching of size $n / 2$, go to Step 2. Otherwise, $a k(G)=0$ and stop. Then $\varnothing$ is the only smallest anti-Kekulé set of $G$.

Step 2. Set $k \leftarrow k+1$. Screen all edge subsets $S$ of size $k$ and let $\mathcal{F}_{k}:=\{S|| S \mid=$ $k$ and $S \subset E(G)\}$. Go to Step 3.

Step 3. Choose an $S$ from $\mathcal{F}_{k}$, apply the BFS algorithm to find a spanning tree of $G-S$. If $G-S$ has no spanning tree, go to Step 4. Otherwise, apply the maximum matching algorithm to $G-S$. If $G-S$ has a maximum matching of size $n / 2$, go to Step 4 . Otherwise, label $S$ as a smallest anti-Kekulé set and go to Step 4.

Step 4. Set $\mathcal{F}_{k} \leftarrow \mathcal{F}_{k} \backslash\{S\}$. If $\mathcal{F}_{k} \neq \varnothing$, return to Step 3. Otherwise, go to Step 5.

Step 5. If there is no labeled edge set, go to Step 2. Otherwise, output all labeled sets and stop.

The screening process in Step 2 takes at most $\left(\begin{array}{c}m \\ k\end{array}\right)$ steps. By Theorem $1.4, k \leq 4$. So the worst case takes $\left(\begin{array}{c}m \\ 4\end{array}\right)$ steps, which is $O\left(m^{4}\right)$ steps. It takes $O(n)$ steps to run BFS algorithm for $G-S$ and $O(\sqrt{n} m)$ steps to find a maximum matching of $G-S$. So for a given $S$, it takes at most $O(\sqrt{n} m)$ steps to determine whether it is an anti-Kekulé set or not. Therefore, the worst case takes $O\left(\sqrt{n} m^{5}\right)$ steps to find all smallest anti-Kekule sets of $G$. Since $G$ is a cubic graph, $m=3 n / 2$. So we have the following result. 
Theorem 4.2. Let $G$ be a connected cubic graph with $n$ vertices. Then it takes $O\left(n^{11 / 2}\right)$ steps to find out all the smallest anti-Kekulé sets of $G$.

\section{References}

[1] N. Blum, A new approach to maximum matching in general graphs, in: M. Paterson (ed.), Automata, Languages and Programming, Springer, Berlin, volume 443 of Lecture Notes in Computer Science, 1990 pp. 586-597, doi:10.1007/bfb0032060, proceedings of the 17th International Colloquium (ICALP 1990) held at Warwick University, England, July 16 - 20, 1990.

[2] J. Cai and H. Zhang, On the anti-Kekulé number of a hexagonal system, MATCH Commun. Math. Comput. Chem. 69 (2013), 733-754, http://match.pmf.kg.ac.rs/ electronic_versions/Match69/n3/match69n3_733-754.pdf.

[3] A. Ceulemans, S. Compernolle, A. Delabie, K. Somers, L. F. Chibotaru, P. W. Fowler, M. J. Margańska and M. Szopa, Electronic structure of polyhedral carbon cages consisting of hexagons and triangles, Phys. Rev. B 65 (2002), 115412, doi:10.1103/physrevb.65.115412.

[4] M. DeVos, L. Goddyn, B. Mohar and R. Šámal, Cayley sum graphs and eigenvalues of $(3,6)-$ fullerenes, J. Comb. Theory Ser. B 99 (2009), 358-369, doi:10.1016/j.jctb.2008.08.005.

[5] T. Došlić, Cyclical edge-connectivity of fullerene graphs and $(k, 6)$-cages, J. Math. Chem. 33 (2003), 103-112, doi:10.1023/a:1023299815308.

[6] J. Edmonds, Paths, trees, and flowers, Canad. J. Math. 17 (1965), 449-467, doi:10.4153/ cjm-1965-045-4.

[7] H. N. Gabow, An efficient implementation of Edmonds' algorithm for maximum matching on graphs, J. ACM 23 (1976), 221-234, doi:10.1145/321941.321942.

[8] H. N. Gabow and R. E. Tarjan, Faster scaling algorithms for general graph-matching problems, J. ACM 38 (1991), 815-853, doi:10.1145/115234.115366.

[9] P. R. Goodey, Hamiltonian circuits in polytopes with even sided faces, Israel J. Math. 22 (1975), 52-56, doi:10.1007/bf02757273.

[10] P. R. Goodey, A class of Hamiltonian polytopes, J. Graph Theory 1 (1977), 181-185, doi: 10.1002/jgt.3190010213.

[11] P. Hall, On representatives of subsets, J. London Math. Soc. 10 (1935), 26-30, doi:10.1112/ jlms/s1-10.37.26.

[12] J. E. Hopcroft and R. M. Karp, An $n^{5 / 2}$ algorithm for maximum matchings in bipartite graphs, SIAM J. Comput. 2 (1973), 225-231, doi:10.1137/0202019.

[13] X. Jiang and H. Zhang, On forcing matching number of boron-nitrogen fullerene graphs, Discrete Appl. Math. 159 (2011), 1581-1593, doi:10.1016/j.dam.2011.05.006.

[14] P. E. John and H. Sachs, Spectra of toroidal graphs, Discrete Math. 309 (2009), 2663-2681, doi:10.1016/j.disc.2008.06.034.

[15] D. J. Klein, Valence bond theory for conjugated hydrocarbons, Pure Appl. Chem. 55 (1983), 299-306, doi:10.1351/pac198855020299.

[16] K. Kutnar, J. Sedlar and D. Vukičević, On the anti-Kekulé number of leapfrog fullerenes, $J$. Math. Chem. 45 (2009), 431-441, doi:10.1007/s10910-008-9416-1.

[17] H. Lü, X. Li and H. Zhang, Np-completeness of anti-Kekulé and matching preclusion problems, 2017, arXiv:1706.09321 [math.CO].

[18] S. Micali and V. V. Vazirani, An $O(\sqrt{|V|} \cdot|E|)$ algorithm for finding maximum matching in general graphs, in: 21st Annual Symposium on Foundations of Computer Science, IEEE Computer Society, 1980 pp. 17-27, doi:10.1109/sfcs.1980.12, proceedings of the 21st Annual 
Symposium on Foundations of Computer Science held in Syracuse, New York, USA, October $13-15,1980$.

[19] J. Petersen, Die Theorie der Regulären Graphs, Acta Math. 15 (1891), 193-220, doi:10.1007/ bf02392606.

[20] Z. Qi and H. Zhang, A note on the cyclical edge-connectivity of fullerene graphs, J. Math. Chem. 43 (2008), 134-140, doi:10.1007/s10910-006-9185-7.

[21] M. Szopa, M. Margańska and E. Zipper, Geometry and topology induced electronic properties of graphene derived quantum systems, Int. J. Theor. Phys. 42 (2003), 1119-1132, doi:10.1023/ a:1025499329135.

[22] S. Tang and H. Deng, On the anti-Kekulé number of three fence graphs, Dig. J. Nanomater. Bios. 6 (2011), 439-443, http: / / www. chalcogen.ro/439_Tang.pdf.

[23] W. T. Tutte, The factorization of linear graphs, J. London Math. Soc. 22 (1947), 107-111, doi:10.1112/jlms/s1-22.2.107.

[24] V. V. Vazirani, A theory of alternating paths and blossoms for proving correctness of the $O(\sqrt{V} E)$ general graph maximum matching algorithm, Combinatorica 14 (1994), 71-109, doi:10.1007/bf01305952.

[25] D. Veljan and D. Vukičević, The anti-Kekulé number of the infinite triangular, rectangular and hexagonal grids, Glasnik Matematički 43 (2008), 243-252, doi:10.3336/gm.43.2.02.

[26] D. Vukičević and T. Došlić, Global forcing number of grid graphs, Australas. J. Combin. 38 (2007), 47-62, https://ajc.maths.uq.edu.au/pdf/38/ajc_v38_p0 47 .pdf.

[27] D. Vukičević and N. Trinajstić, On the anti-Kekulé number and anti-forcing number of catacondensed benzenoids, J. Math. Chem. 43 (2008), 719-726, doi:10.1007/s10910-006-9223-5.

[28] Q. Yang, D. Ye, H. Zhang and Y. Lin, On the anti-Kekulé number of fullerenes, MATCH Commun. Math. Comput. Chem. 67 (2012), 281-288, http://match.pmf.kg.ac.rs/ electronic_versions/Match67/n2/match67n2_281-288.pdf.

[29] R. Yang and H. Zhang, Hexagonal resonance of (3,6)-fullerenes, J. Math. Chem. 50 (2012), 261-273, doi:10.1007/s10910-011-9910-8.

[30] D. Ye, On the anti-Kekulé number and odd cycle transversal of regular graphs, Discrete Appl. Math. 161 (2013), 2196-2199, doi:10.1016/j.dam.2013.03.014.

[31] D. Ye, Z. Qi and H. Zhang, On $k$-resonant fullerene graphs, SIAM J. Discrete Math. 23 (2009), 1023-1044, doi:10.1137/080712763.

[32] D. Ye and H. Zhang, 2-extendability of toroidal polyhexes and Klein-bottle polyhexes, Discrete Appl. Math. 157 (2009), 292-299, doi:10.1016/j.dam.2008.03.009.

[33] H. Zhang and S. Liu, 2-resonance of plane bipartite graphs and its applications to boronnitrogen fullerenes, Discrete Appl. Math. 158 (2010), 1559-1569, doi:10.1016/j.dam.2010.05. 012.

[34] H. Zhang, D. Ye and W. C. Shiu, Forcing matching numbers of fullerene graphs, Discrete Appl. Math. 158 (2010), 573-582, doi:10.1016/j.dam.2009.10.013.

[35] Q. Zhang, H. Bian and E. Vumar, On the anti-Kekulé and anti-forcing number of cata-condensed phenylenes, MATCH Commun. Math. Comput. Chem. 65 (2011), 799806, http://match.pmf.kg.ac.rs/electronic_versions/Match65/n3/ match65n3_799-806.pdf. 